\title{
Interactive comment on "Weichselian permafrost depth in lowland Europe: a comprehensive uncertainty and sensitivity analysis" by $\mathrm{J}$. Govaerts et al.
}

D. Kitover (Referee)

d.c.kitover@vu.nl

Received and published: 14 June 2016

The discussion paper "Weichselian permafrost depth in lowland Europe: a comprehensive uncertainty and sensitivity analysis by Govaerts et al. 2016 explores potential permafrost depth give a range of uncertainty in The Netherlands. This investigation is compelled by the underlying possibility for future storage of nuclear waste.

General Comments:

Overall, this work is understandable and presents a methodological approach to assign estimates given a large range of uncertainty. However, there are some areas which seems to give superfluous information and unnecessary details while some other sec- 
tions require more explanation and greater detail.

I think it is important to include more detail on the parameters of the model and subsurface characteristics and how they vary. At one point, thermal conductivity is said to be averaged. However, from the model description they seem to change as a function of frozen/unfrozen. It is not clear what varies spatially, either from polygon to polygon, or vertically through the 1-d mesh. The porosity is mentioned plenty of times but is it stated that the model assumes full saturation and that the porosity equals water content? If this study is to be added into the inventory of past permafrost estimates, the understanding of the model construction, assumptions, initialization, and parameter settings should be very clear. This allows future research to make fair comparisons.

The description of the uncertainty analysis on page 10 should be condensed. It distracts the reader.

\section{Specific Comments:}

P2, L1-2: This first sentence needs a reference.

P2, L11: "periglacial conditions will reappear". Reappear where? At current state, periglacial conditions are existing already. Please be more specific at this kind of suggestion.

P5, L32: What do you mean by "conservatively" neglected?

P6, L13: This is the point at which the vertical description of the model is presented and it is overlooked. What is the depth and how does the mesh reflect the varying subsurface characteristics? This is confusing with your description on P6, L31. How deep is the lower boundary?

P6, L22: "The input temperature is held constant for the entire country". Do you mean for initialization or a spin-uo?. Surely, the temperature forcing changes temporally and spatially so what is this sentence describing? I suppose the intial setup? And what is the input temperature?

Printer-friendly version

Discussion paper 
P6, L30 to P7, L2: I don't understand these dimensions and the following descriptions. This section should be described with improved clarity.

P7, L14-15: REGIS model should have a reference.

P8, L3: you may want to replace "input" here, and in other spots in manuscript. with the term "forcing".

P9, L13: ..as soil input data.." is vague. Maybe write surface temperature data or land temperature forcing..

P9, L17: "shielding effect of snow and temperature" does not make sense

P9, L30: "average thermal conductivity values". Does this mean there is only one thermal conductivity value per polygon or just for the purpose of calculating a thermal gradient?

P12, L3-5: The T1 to T26 are simply time intervals or snapshots of the transient simulation? Is this a variable you control for? A table should be provided that relates the $T$ variables with their time interval.

P14, L9: You state that a higher parameter will cause a larger permafrost depth but how is this possible if for example, the geothermal heat flux parameter is high, which in turn would cause less permafrost to develop?

P14, L1-9: If you state earlier that you use SRC for this study (P12, L20), why are you discussing PCC?

P14, L16: How can you say that geothermal heat flux is the main driving force of degradation? Is it not the temperature forcing at the surface? Is this accounted for by the R2? If you find that the geothermal heat flux accounts strongly for permafrost warming MORE than the surface temperatures, this is an important finding that should be clarified and explained.

P15, L9-12: This is an important point but should be referenced by earlier studies which 
discuss how surface forcings penetrate deeper into soil and the time frame associated with the lag.

P15, L15 and further: Although you are comparing your estimates at $50 \%$ frozen this is probably not the case with the other studies, which assume $100 \%$ frozen and then this depends on what their freezing algorithm was, what temperature etc.

Almost all the figure and/or figure captions should be improved: Figure 1: Make sure all 17 polygons are represented and listed - my count was off. Figure 2: "mid-depth" porosity, is this half-way ? Figure 4: Consider making y-axis scale same for both figures to more easily compare the two sites. Figure 6: X-axis "Distance" from what? Maybe put a little insert to illustrate the distance. Figure 7: This figure caption does not explain what the percentile is of? The secondary $\mathrm{x}$-axis should have a unit associated with it (\% I think). Figure 8: Both figures should align even on top of each other for more illustrative comparison. Permafrost thickness should be also illustrated with the curved, perhaps in a third figure below.

Technical corrections:

Make sure at first mention of acronyms that they are defined. For example...DGM

P1 L10 remove 'being'

P3 L10 replace 'so-called' with 'kind of'

P3 L24 replace "the thorough" with "a more thorough"

P5 L20 - L30: Check consistent use of units Figure 1: Are all the structural elements (17) listed in the figure caption and on the map? Are the names consistently referenced?

Printer-friendly version

P8, L33: Remove "including"

P8, L33: should read “. . future glacial colder than..” 
P8, L20: remove "conservative" and write "..the model neglects subsurface hydrology such as the vadose zone and groundwater flow. During very cold..."

P11, L4: remove "exhaustively"

P11, L14: correct as such "tables of collected results produced by Matlab can then be directly analysed to calculate and plot the.."

P11, L16: Would sound better to begin sentence "Matlab was then used again to compute"

P11, L 20: Are you just being redundant from line 17 by again stating you performed 1000 realizations?

P12, L2: extra period

P12, L5: comma before which

P12, L12: remove "(and other local SA methods)"

P12, L29: should be written "..are shown as a function of time.."

P14, L21: remove "away"

P16, L8: don't end sentence with a preposition such as "to"

P17, L3: "error" is not a good word here. Consider a less negative word such as discrepancy or unknowns

Interactive comment on The Cryosphere Discuss., doi:10.5194/tc-2016-54, 2016. 